\title{
Research on Improved Fuzzy Optimization Routing Problem in WSNs Based on Genetic Ant Colony Algorithm
}

\author{
Xiaoguang $\mathrm{Li}^{1 *}$, Guanghong $\mathrm{Li}^{2}$, Songan Zhang $^{3}$ and Qiang Yuan ${ }^{3}$ \\ ${ }^{1}$ Department of Electrical Engineering and Automation, Luoyang Institute of \\ Science and Technology, Luoyang 471023, China \\ ${ }^{2}$ Project Practice Centre, Luoyang Institute of Science and Technology, Luoyang \\ 471023, China \\ ${ }^{3}$ Luoyang Urban Planning \& Architecture Design Research Institute Company \\ Limited, Luoyang 471003, China \\ lylglxg@163.com
}

\begin{abstract}
The combination of traditional ant colony algorithm in solving the optimization process to consume a large amount of time, easily falling into local optimal solution and convergence is slow and other disadvantages, while also generating a lot of useless redundant iterative code, operation efficiency is low. Therefore, ant colony optimization algorithm is proposed. The algorithm based on genetic algorithm has the ability to search the global ant colony algorithm also has a parallel and positive feedback mechanisms. Changes in the use of genetic algorithm selection operator, crossover operator and mutation operator action to determine the distribution of pheromone on the path, the ant colony algorithm for feature selection using support vector machine classifiers for evaluating the performance characteristics of the feedback sub-Variorum And by changing the pheromone iteration, parameter selection and increase the local pheromone update feature nodes guided the re-combination. The algorithm uses probability expectation values are obtained to meet under the conditions with minimal sensor nodes, and gives the optimal coverage and connectivity probability models and reasoning. The experimental results show that, the algorithm can not only use the least nodes complete the effective target area to be covered, and in reducing the network energy consumption is also greatly improved, simultaneously reduces the cyber source configuration, improve the network life cycle.
\end{abstract}

Keywords: Wireless sensor networks; Genetic ant colony algorithm; Network lifetime

\section{Introduction}

Ant colony algorithm was put forward by Dorigo, Maniezzo and Colorni in 1991, which is the original of the ACO algorithm. The algorithm is applied to the Traveling salesman Problem early ('ll Salesm an Problem, TSP) scheduling Problem, assignment Problem, Problem solving, etc. Feature selection problem is a NP problem, selection methods are divided into two categories: the Filter and Wrappe. Its main idea is to search a close to the approximate optimal solution. Process is characteristic of the Filter class mainly adopts specific independent inductive learning algorithm, and its main characteristic is to the whole path search speed faster, but the algorithm in the application process.

A precocious phenomenon, make the low accuracy; Wrapper class also has the characteristic of inductive learning algorithm and its performance as the characteristics of the evaluation and selection standard, its main characteristic is algorithm accuracy is higher, but each to a subset of the evaluation to the restructuring of the calculation, the 
computational complexity is high, not suitable for large-scale data search and calculation. Combined with the Filter and Wrapper is two complementary mode, a combination of both for the study of ant colony algorithm provides a larger value [1]. Literature [2] is will be introduced to the weights of the pheromone updating process, improve the global search ability by repeated iteration to obtain the shortest path. Reference [3] is used by the transition probability and pheromone distribution estimation algorithm combining produce new individuals to improve the convergence speed and global search ability to inhibit earliness and defects [4] proposes a genetic ant colony algorithm, the idea is to use genetic algorithm to search the global first, and then use ant colony algorithm for the solution of optimization is to achieve the objective of the optimal solution.

Improved genetic ant colony algorithm, this paper USES the Filter and Wrapper class characteristics, together with mutual information as the heuristic function, the use of ant colony algorithm to dynamic search feature subset, and combining classifier classification effect as evaluation criteria, based on the characteristics of the node classification precision guidance pheromone update, based on the genetic algorithm to produce a more optimal solution of dynamic change crossover operator and mutation operator at the same time, the solution of the optimal path pheromone intensity, and then according to the ant colony algorithm after completion of a traversal of path pheromone update and local pheromone iteration, global update strategy to enhance the ability of global optimization, speed up the convergence speed, the system improves the searching efficiency and avoid the premature phenomena in the process of iteration, restrain redundant code, makes more accurate precision effectively inhibit the occurrence of the phenomenon of premature convergence, improves the genetic ant colony algorithm in the limitations of global convergence and improves the convergence speed, the experimental results show that the algorithm has higher efficiency.

In addition, with the increase of the sensor nodes and coverage region changes, the complexity of the algorithm will become larger, and thus computational efficiency will reduces [5-7]. The coverage configuration protocol proposed by reference [8-10] is to judge the distributive node functions qualification using the local node location information, which is when the communication radius is greater than or equal to 2 times the sensing radius and the network k-cover the given convex region in the region [11, 12], the network is k-connected, thereby promoting its agreement to meet the multi-coverage requirements. LEACH protocol has been improved in reference [13-15]. The main idea is: clustering routing algorithm uses periodic random selection of cluster head node to balance the node energy consumption and to achieve the purpose to extend the network lifetime [16-18]. Under the premise of maintaining a certain coverage and connectivity rate, in the operation of each data retrieval collected by the sensor nodes [19], a subset of a sensor node is selected to collect data and to realize nodes subsets dynamic scheduling conversion mechanism [20,21], and thus achieve the optimal choice for each subset of data acquisition, thereby optimizing the number of nodes, improving network stability and extending the lifetime of wireless sensor networks [22, 23]. Finally, the number relations of nodes needed to be deployed in coverage and connectivity is given under the condition whether there is influence of edge node.

\section{Problem Description}

The coverage and connectivity this paper studies is based on local positioning algorithm and has the following basic assumptions:

Assumption 1: The covering radius and communication radius of each node showed a disc-shaped.

Assumption 2: the specific location information of its own node is obtained through some localization algorithm, such as: the RSSI location algorithm, the Euclidean 
positioning algorithm, without the participation of large-scale physical communications equipment.

Assumption 3: All sensor nodes are randomly and uniformly deployed in square in side length $l$ monitoring region $\Omega$ with consideration of the boundary of deployment region.

Assumption 4: The perception of each node is much smaller than the entire network coverage region, and all nodes are isomorphic and initial energies are equal.

Definition 1: Set up a two-dimensional plane $E$, the coordinates of the nodes $s_{i}\left(x_{i}, y_{i}\right)$, the coordinates of the target $t_{k}\left(x_{k}, y_{k}\right)$, the Euler distance between the nodes $s_{i}$ and $t_{k}$ is less than $s_{i}$, perception radius $r_{s}$ : that is $E S_{i}=\left\{t_{k} \mid t_{k} \in E^{2}, D\left(s_{i}, t_{k}\right) \leq r_{s}\right\}$, said point $t_{k}$ is covered by $s_{i}$.

Definition 2: for a given target set $T=\left\{t_{1}, t_{2}, t_{3} \cdots t_{k}\right\}$ and the sensor node set $V=\left\{v_{1}, v_{2}\right.$, $\left.v_{3} \cdots v_{n}\right\}$, in a time slot, if the target set $T$ in any goal $t_{k}$ is covered by at least one node within node set $V$, then the target set $\mathrm{T}$ is full coverage.

Definition 3: suppose that nodes $s_{i}, s_{j}$, the target regions they cover are $C_{i}$ and $C_{j}$, and $C_{i} \cap C_{j} \neq \varnothing$, so nodes $s_{i}$ and $s_{j}$ are coverage connected. Suppose that $T$ is a $m$ nodes set randomly distributed in the target region, $E$ is the set of edges of the network diagram, indicating that the $e_{i j}=1$ positional relationship in $e_{i j} ; e_{i j}$ represents the positional relationship of node $s_{i}$ and the target node $t_{j}$. When $e_{i}=1$ when and only when the Euclidean distance of target node $t_{j}$ and the node $s_{i}$ is less than or equal to the perception radius $r_{i}$, otherwise $e_{i}=0 . W=\left\{w_{1}, w_{2} \cdots w_{n}\right\}$ is the initial energy set of sensor nodes; $W$ is in normal distribution; the $w_{i}$ represents initial energy of sensor node $s_{i} ; w_{i}$ is the maximum energy in the process of node work.

Definition 4: In the monitoring region any point at least at the same time located in the perception range of $K$ nodes is known as the $K$ re-cover. That is $A_{k} \in \prod_{i=c}^{c+k} A s_{i}$, where $1 \leq \mathrm{c} \leq \mathrm{n}$.

Definition 5: coverage rate of a point $s_{i}$ in coverage region is as follows:

$$
p\left(s_{i}, s_{j}\right)= \begin{cases}0 & \text { if } R_{s} \leq d\left(s_{i}, s_{j}\right) \\ e^{-\varepsilon d} & \text { if }\left(R_{s}-R_{e}\right)<d\left(s_{i}, s_{j}\right)<R_{s} \\ 1 & \text { if } d\left(s_{i}, s_{j}\right) \leq\left(R_{s}-R_{e}\right)\end{cases}
$$

Of which: $d=d\left(s_{i}, s_{j}\right)-\left(R_{s}-R_{\mathrm{e}}\right), \varepsilon$ is physical parameters of the sensor node; $R_{\mathrm{e}}$ represents the monitoring dynamic parameters of the sensor nodes; $d\left(s_{i}, s_{j}\right)$ represents the Euclidean distance between the sensor nodes $s_{i}, s_{j}$; When the coverage $p\left(s_{i}, s_{j}\right)$ of a node $s_{i}$ meets $d\left(s_{i}, s_{j}\right) \leq\left(R_{s}-R_{e}\right)$, then the node is detected.

In the balance accelerate convergence and reduce the stagnation is one of the difficulties of the current research of ant colony algorithm. The algorithm as the search space as large as possible, to find the possible solution of the optimal solution space; Current at the same time, should make full use of community with the effective information, adapt algorithm focuses on those who may have a higher value of individual space, with large probability convergence to the global optimal solution. With dynamic weighting method, based on the ant solution quality is different, give different weights. According to different weight, the characteristics of ants by pheromone adaptive update. Therefore, to determine ant colony is gathered to one or a few solutions or scattered among the search space. Can through the calculation of the average of the iterative solution of all the characteristics of the subset classification error rate and the iterative solution of the minimum feature subset classification error rate to determine the differences between degrees of polymerization of ant colony. Can be concluded if the 
whole ant colony is aggregation, then the difference is smaller than relatively decentralized ant colony. So can be the difference between the two as a measure of whether ant colony convergence.

$$
E_{\text {avg }}=\frac{1}{n} \sum_{k=1}^{n a} E_{k}
$$

Ants in the iteration of the structure of the solution of the feature subset classification error rate, when updating pheromone, for less than ants gives a relatively large weight, the less you give weight, the greater the for more than or equal to the ant to give a relatively small fixed weights. So the ants in the current iteration of the selected feature on the release of pheromones as follows:

$$
\Delta \tau\left(f_{i}\right)=\left\{\begin{array}{l}
Q \lambda_{k} / E_{\text {avg }} \\
0
\end{array}\right.
$$

One type is obtained under the weight of:

$$
\lambda_{k}=\left\{\begin{array}{l}
\left(E_{\text {avg }}-E_{k}\right) /\left(E_{\text {avg }}-E_{\text {min }}\right) \\
0 \quad E_{k} \geq E_{\text {avg }}
\end{array}\right.
$$

Can see the closer, the weights of lambda approximates to 1 . According to the formula (4) the structure characteristics of the calculation of each iteration the ant, again according to individual ants tectonic characteristics of solution and the differences between the corresponding pheromone for change. So you can increase the diversity of the solution space, improve the global search ability of ant colony, to avoid local convergence too fast, at the same time also won't reduce the search speed of ant colony. In the ant colony algorithm, the ant principle of selecting the next node structure solution and pheromone update rules of ant colony can search to the optimal solution play a decisive role.

Usually, coverage directly reflects the extent that the objectives are concerned, the concerned target node region having higher coverage, taking into account the functional relationship between sensor node $p$ in the region II expectations and coverage region, shown in Figure 1.

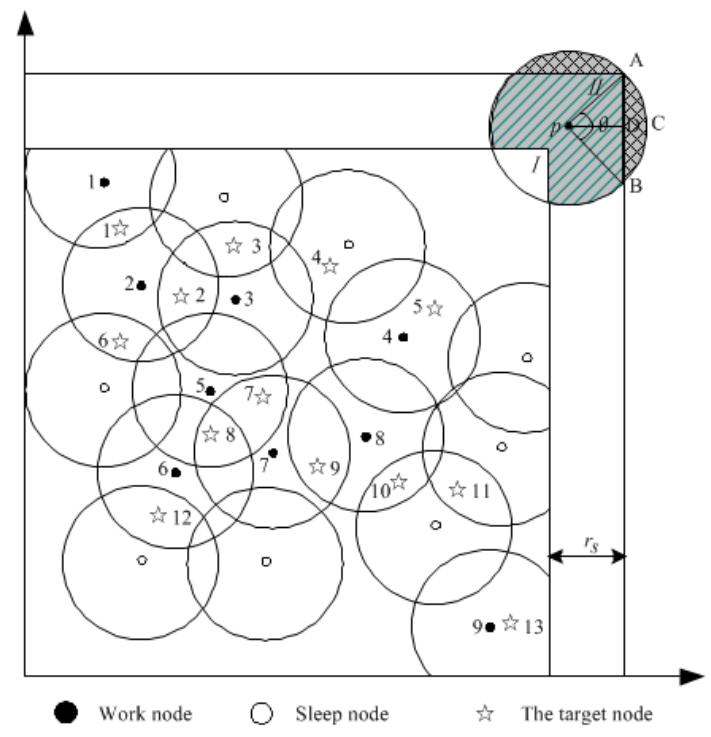

Figure 1. Diagram of Connective Coverage of Target Region

Now take the analysis of Figure 1 as the example. The square region 1 is divided into I and II two parts. Randomly deploy the nodes in the monitoring region to construct a finite set $S$ with the coverage region of each node being $E(C)$, so that the coverage 
probability of each node is $E(C) / \Omega$. When the nodes set is empty, the network coverage of the n deployed nodes will be $P(S)=(1-E(C) / \Omega)^{n}$. Thus the network nodes probability value has been obtained in the situation that collective $\mathrm{S}$ is not an empty set.

$$
P(S)=1-(1-E(C) / \Omega)^{n}
$$

When the number of nodes $n \rightarrow \infty, \lim _{n \rightarrow \infty} E(P(S))=1$, which indicates that the number of nodes is large enough, this coverage region is completely covered[10]. Considering the boundary effect in solving the node coverage region and expectations, because the square region is divided into region I and II, based on the concept of expectation value in probability, expectation value of the coverage of network nodes can be obtained:

$$
E(C)=P\left(\Omega_{I}\right) E\left(C_{\Omega_{I}}\right)+P\left(\Omega_{I I}\right) E\left(C_{\Omega_{I I}}\right)
$$

Among them, $P\left(\Omega_{I}\right)$ and $P\left(\Omega_{I I}\right)$, respectively represents the probability values of the randomly deployed nodes in region I and II. $E\left(C_{\Omega_{I}}\right)$ and $E\left(C_{\Omega_{I I}}\right)$ denote the corresponding expectations of coverage. From the even distribution function of the random deployment of sensor nodes, then gets:

$$
P\left(\Omega_{I}\right)=\left(l-r_{s}\right)^{2} / l^{2}, P\left(\Omega_{I I}\right)=2 r_{s}\left(l-r_{s}\right) / l^{2}
$$

Assume that node $p$ is in the region I, its coverage being completely contained, so the coverage expectation is:

$$
E\left(C_{\Omega_{I}}\right)=\pi r_{s}^{2}
$$

When a node $p$ is in region II, the region should equal to its sensing region of the circumference subtracting bow region $S_{A C B D} . A$ and $B$ is the intersection of the sensing circle of node $p$ and network boundaries, whose angle $\theta$ is the central angle formed by the node $p$ and A, B. i.e. $\angle A p B=\theta, \theta=2 \arccos y / r_{s}$ so:

$$
\begin{aligned}
E\left(C_{\Omega_{I I}}\right) & =2 \int_{0}^{l-r_{s}} \frac{1}{2} r_{s}^{2}(2 \pi-\theta+\sin \theta) d x \int_{0}^{r_{s}} \frac{1}{2\left(l-r_{s}\right) r_{s}} d y \\
& =\frac{r_{s}}{2\left(l-r_{s}\right)} \int_{0}^{l-r_{s}}(2 \pi-\theta+\sin \theta) d x \int_{0}^{r_{s}} d y
\end{aligned}
$$

\section{Hybrid Genetic Algorithm}

\subsection{Ant Colony Algorithm}

Ants in the process of search are always hoped to meet the first select the evaluation function of the node. When the initial, all nodes of the pheromone is consistent, need more inspiration function to guide the ant? In basic TSP problem, the heuristic function is defined as the reciprocal of the distance between the two cities, in the search for characteristics of the node is not applicable. Due to the Filter class feature selection method, the mutual information between features and categories can better reflect the characteristics of the importance of inspiration function for that we will each feature nodes is defined as the characteristic and the category of mutual information, as shown below:

$$
\eta_{f i}=I\left(f_{i}: \mathrm{C}\right)
$$


Initial each ant randomly placed on the characteristics of a node, starting from the starting point a feature based on state transition rules of the selection. After the ants construct the solution, for all the characteristics of the solution of training classification respectively, get the classification error rate, and assess its advantages and disadvantages. Ant select features take according to the formula (10).

$$
\begin{gathered}
s=\left\{\begin{array}{l}
\arg \max \left\{\tau(u)^{\alpha} * \eta(u)^{\beta}\right\}, \text { if }\left(q<q_{0}\right), u \in \text { allowed }_{k} \\
p(s), \text { otherwise, } s \in \text { allowed }_{k}
\end{array}\right. \\
p(s)=\frac{[\tau(s)]^{\alpha *[\eta(s)]^{\beta}}}{\sum_{u \in \text { allowed }_{k}}[\tau(u)]^{\alpha} *[\eta(u)]^{\beta}}
\end{gathered}
$$

Among them: alpha, beta, said the relative importance of the pheromone and the heuristic information; Refers to the current ant search all optional feature set; Said the ant the currently selected features; With the features of adjacency; Ants said the next step allows you to select all of the nodes, the list of records through the current ant nodes, when all nodes are written to the tabu table, ant to complete a cycle, the route is a solution of the problem by. Is evenly distributed in the interval $[0,1]$ is a random variable, the rules used to determine the relative importance of two parts, namely the ants selection can make the greatest feature of the pheromone and the heuristic information of the product and the most ants choose to have the characteristics of the transition probability of the two parts. To ensure ants use past information also have the ability to develop new search space at the same time, to prevent too fast convergence to local optimum.

After moving from node to node on the path of the pheromone update according to the formula (13)

$$
\tau_{i j}(t+1)=(1-\xi) \tau_{i j}(t)+\xi \Delta \tau_{i j}(t)
$$

Among them, is the initial value of information? So can effectively avoid falling into local optimum, reduced has chosen a path to the selected probability again, so as to effectively avoid the ants converge to the same path, and improve the global search ability of the algorithm.

Ants search focuses in the current cycle so far to find the shortest path field area, global update rule is in after all the ants have completed their path to perform [13], update the pheromone formula is

$$
\begin{gathered}
\tau_{i j}(t+1)=\left(1-\xi \tau_{i j}(t)\right) \tau_{i j}(t)+\xi \tau_{i j}(t) \Delta \tau_{i j}(t) \\
\Delta \tau_{i j}^{k}= \begin{cases}\frac{Q}{L_{\min }} & S(i, j) \in L_{\min } \\
-\frac{Q}{L_{\max }} & S(i, j) \in L_{\max } \\
0 & \text { otherwise }\end{cases}
\end{gathered}
$$

A pheromone volatilization coefficient, such as to do some change, it can prevent the increase of the pheromone concentration on the path of the infinite, can reduce the possibility of trapped in local optimum, and thus accelerate the convergence speed. Ants are through the shortest and the longest path. In the early stages of the algorithm, the ant 
walk through every path has a chance to get the amount of information updates, ant chooses the path of the corresponding probability can be improved. With certain path pheromone accumulation, reach a certain number in cycles, reduced the optimal path with the worst side of the path between the amounts of information gap, and so that the ant search behavior has focused on the optimal path.

Reasonable parameter is set to improve the convergence speed of the system, enhanced search capabilities for global; effectively inhibit the premature emergence of stagnation. When there is $\alpha$ and $\beta$, too big or too hours adjusted formula as:

$$
\begin{aligned}
& \alpha= \begin{cases}\kappa \alpha & \tau_{i j}(t+1) \leq \tau_{i j}(t) \\
\alpha & \text { otherwise }\end{cases} \\
& \beta= \begin{cases}\kappa \beta & \tau_{i j}(t+1) \geq \tau_{i j}(\mathrm{t}) \\
\beta & \text { otherwise }\end{cases}
\end{aligned}
$$

$k$ is the scaling factors. $k=\tau_{i j}(t) / \tau_{i j}(t+1)$ Through dynamic $k$ adjustment of the scaling factors $\alpha$ and $\beta$ can be completed to ensure the validity of the algorithm. $\rho$ are pheromones volatile factors, $(1-\rho)$ was residual factor pheromone. $\rho$ is too large, the previous search and path was again selected probability increases, it will result in random and the drop in overall search capability. Once an hour, $\rho$ increased global search capacity while reducing the rate of convergence.

\subsection{Adaptation Function}

Fitness is the individual groups the opportunity to choose the only certainty of life indicator. Adaptive function is directly determines the evolution of group behavior. For minimizing issues, to establish function $f(x)$ and $g(x)$ objective function of mapping relations:

$$
f(x)= \begin{cases}c_{\max }-g(x) & g(x)<c_{\max } \\ 0 & \text { otherwise }\end{cases}
$$

$c_{\max }$ is an input or a theoretical maximum value. By adapting function on target selection and some kind of evolutionary process control functions transformation in order to formulate an appropriate selection policy, evolution of hybrid Ant Colony algorithm for maximum capacity and the best search results.

Theorem1: equipment information $\tau_{i j}(t)$ global maximum value $\tau_{\max }$ for $\forall \tau_{i j}$ type:

$$
\lim _{t \rightarrow \infty} \tau_{i j}(t) \leq \tau_{\max }=\frac{1}{\rho} g\left(s^{\prime}\right)
$$

Proof: if after each iteration, an arbitrary path isolated section of the amount of information on the $(i, j)$ not more than $g\left(s^{\prime}\right)$. Obviously, in the first iteration, the maximum possible amount of information for $(1-\rho) \tau_{0}+g\left(s^{\prime}\right)$; after a second iteration, is $(1-\rho)^{2} \tau_{0}+(1-\rho) g\left(s^{\prime}\right)+g\left(s^{\prime}\right)$ followed by analogy. Therefore, due to the volatile pheromone, in the $t$ iterations, the amount of information of the upper limit value is: $\tau_{i j}^{\max }(t)=(1-\rho)^{t} \tau_{0}+\sum_{i=1}^{t}(1-\rho)^{t-i} g\left(s^{\prime}\right)$

Thus, when $\rho \subset(0,1)$, and will eventually converge to: $\tau_{\max }=\frac{1}{\rho} g\left(s^{\prime}\right)$ 


\section{Evaluation and Simulation}

Table 1. Working Network Nodes

\begin{tabular}{|c|c|c|c|c|c|}
\hline NO. & x-coordinate/km & Y-coordinate/km & Number & Time/h & completed/h \\
\hline 1 & 3 & 42 & 3 & $(0.78,0.90,1.08)$ & 2 \\
\hline 2 & 16 & 48 & 2 & $(0.78,0.90,1.08)$ & 2 \\
\hline 3 & 19 & 69 & 36 & $(0.78,0.90,1.08)$ & 5 \\
\hline 4 & 37 & 53 & 5 & $(0.78,0.90,1.08)$ & 2 \\
\hline 5 & 49 & 64 & 5 & $(0.78,0.90,1.08)$ & 4 \\
\hline 6 & 55 & 79 & 5 & $(0.78,0.90,1.08)$ & 3.5 \\
\hline 7 & 47 & 87 & 4 & $(0.78,0.90,1.08)$ & 3 \\
\hline 8 & 40 & 89 & 9 & $(0.78,0.90,1.08)$ & 4 \\
\hline 9 & 41 & 9 & 2 & $(0.78,0.90,1.08)$ & 3.5 \\
\hline 10 & 47 & 3 & 8 & $(0.78,0.90,1.08)$ & 4 \\
\hline 11 & 45 & 16 & 25 & $(0.48,0.60,0.78)$ & 4 \\
\hline 12 & 54 & 3 & 9 & $(0.48,0.60,0.78)$ & 2 \\
\hline 13 & 55 & 21 & 3 & $(0.48,0.60,0.78)$ & 3 \\
\hline 14 & 61 & 30 & 2 & $(0.48,0.60,0.78)$ & 3.5 \\
\hline 15 & 64 & 52 & 31 & $(0.48,0.60,0.78)$ & 4 \\
\hline 16 & 72 & 45 & 9 & $(0.48,0.60,0.78)$ & 5 \\
\hline 17 & 74 & 31 & 7 & $(0.48,0.60,0.78)$ & 4 \\
\hline 18 & 85 & 60 & 18 & $(0.48,0.60,0.78)$ & 5 \\
\hline 19 & 88 & 66 & 8 & $(0.48,0.60,0.78)$ & 2 \\
\hline 20 & 93 & 62 & 2 & $(0.48,0.60,0.78)$ & 3 \\
\hline
\end{tabular}

Table 2. Sink Nods

\begin{tabular}{|c|c|c|c|}
\hline NO. & x-coordinate $/ \mathrm{km}$ & Y-coordinate $/ \mathrm{km}$ & Members \\
\hline 1 & 30 & 28 & 2 \\
\hline 2 & 75 & 80 & 1 \\
\hline 3 & 12 & 60 & 2 \\
\hline 4 & 38 & 77 & 2 \\
\hline 5 & 62 & 13 & 3 \\
\hline 6 & 68 & 33 & 2 \\
\hline 7 & 87 & 49 & 3 \\
\hline 8 & 95 & 97 & 1 \\
\hline
\end{tabular}

The algorithm parameters are as follows: $n u m=100 ;$ MAXGEN $=300 ; \quad \delta_{1}=0.9$; $\delta_{2}=1.2 ; \alpha=0.9 ; \beta=1 ; \omega=0.8 ; q=20$; Crossover rate is 0.9 ; mutation rate is 0.05 .

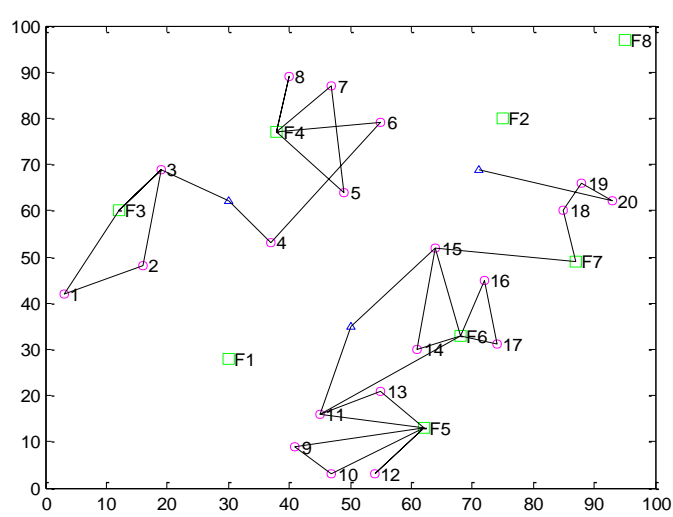

Figure 2. Calculation Results 


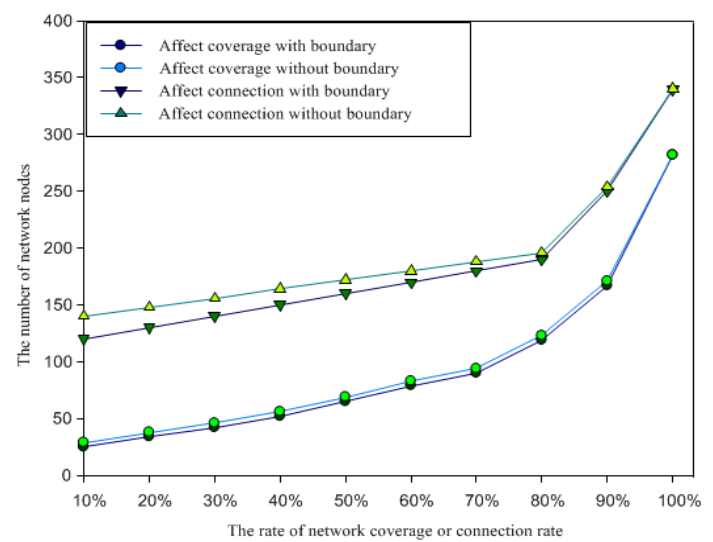

Figure 3. Curves of Network Coverage Rate

Figure 3 reflects the number of nodes required to be deployed to achieve different coverage and connectivity rates without the boundary influence. Compared with the boundary influence, the number of nodes deployed increases slightly, and with the increase of nodes, node density will become larger, so the boundary influence becomes lower.

The algorithm and LEACH protocol comparison experiment, validation in the same round number, the network energy consumption contrast conditions, as shown in Figure 4:

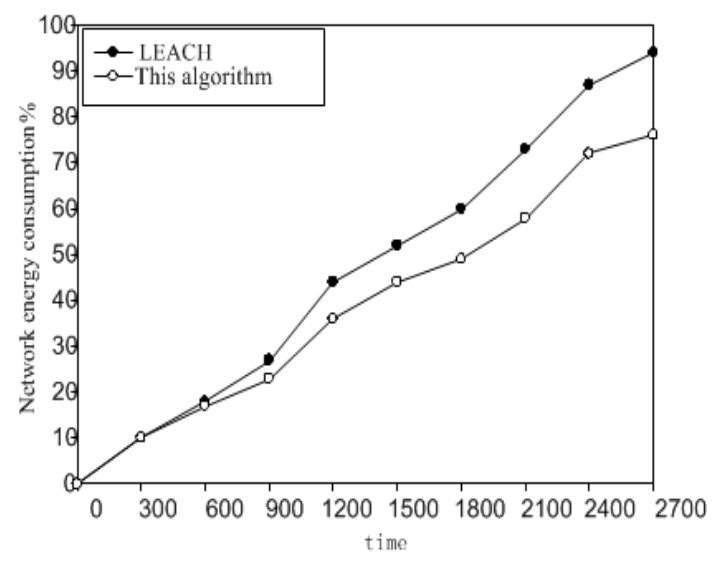

Figure 4. Network Energy Diagram

From the Figure 4 can be seen, in the initial moments, two algorithms of the network energy consumption approximately equal; but as time goes on, the algorithm of the network energy consumption less than LEACH protocol of energy consumption, and this algorithm to the subsequent time tends to be stable, in the entire network cover cycle, this algorithm can effectively save the sensor node energy, prolong the network life cycle.

In second cases, using this algorithm with PEAS algorithm in coverage on the comparison experiment, the coverage rate of $99 \%$ that is completely covered, the experimental data are in 100 simulation data by extracting the average value, as shown in Figure 5: 


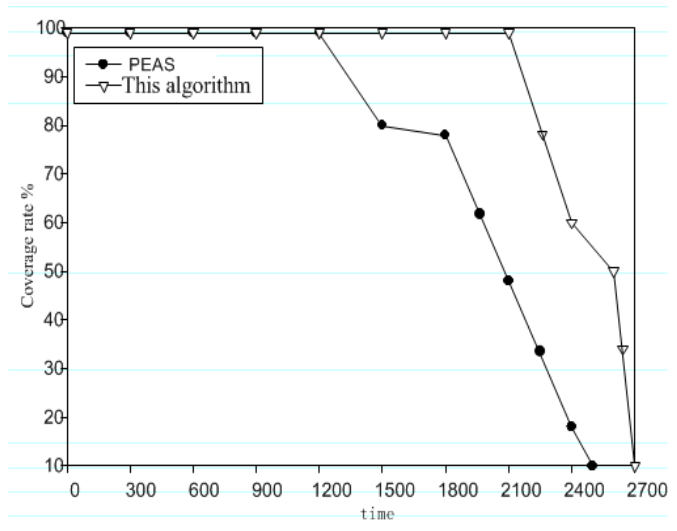

Figure 5. Different Rounds of the Network Coverage

From seeing the Figure 5, as time progresses, two algorithms of coverage has declined. The initial phase, two algorithms of coverage, but in $t=1500$, PEAS algorithm coverage declined more obvious, the algorithm still maintain higher coverage. In the same node under the effect of this algorithm, coverage was significantly higher than that of PEAS algorithm, to verify the effectiveness of this algorithm.

\section{Conclusion}

Improvement, based on the heuristic function, information update strategy, crossover, and selection strategy to optimize the solution value, to a certain extent, inhibit the phenomenon of premature convergence and improve the global searching ability, to avoid falling into local optimal solution effectively, accelerate the convergence speed, In view of the network coverage and connectivity of the random deployment of nodes for wireless sensor network, the relation model of the sensor node and the target node is provided. Considering the boundary influence, coverage and connectivity probability model is provided. It can simplify the computational complexity of network coverage and connectivity to improve the efficiency of the algorithm execution, and thus solve the number of nodes required to deploy more accurately meeting certain network coverage and connectivity rate requirements. Finally, the correctness of the theoretical solution and validity of the algorithm are verified by the simulation results. The main work thereafter is the study of the effective coverage of the irregular region and the entire network energy savings based on sensor network.

\section{Acknowledgments}

The authors wish to thank Science of Technology Research of Foundation Project of Henan Province Education Department under Grant Nos.2014B520099; Natural Science and Technology Research of Foundation Project of Henan Province Department of Science under Grant Nos. 132300410023; Youth Fund Project of Luoyang Institute of Science and Technology Nos. 2013QZ02.

\section{References}

[1] C. G. Qiang, J. L. Min and X. Z. Yi, "Design of Ant Colony Algorithm based Fuzzy Classification System", Fuzzy Systems and Mathematics, vol. 22, no. 4, (2008), pp. 87-98.

[2] F. Z. Hong and X. Z. Ben, "A Hybrid Ant Colony Algorithm for Solving TSP", Journal of Engineering Mathematics, vol. 29, no. 4, (2012), pp. 35-39.

[3] D. Dong and X. Liao, "Distributed coverage in wireless Ad Hoc and sensor networks by topical graph approaches", IEEE Transactions on Computers, vol. 61, no. 10, (2011), pp. 1417-1428.

[4] L. Yin and M Liang, "Fuzzy Ant Colony algorithm and its Application in TSP", Mathematics in Practice and Theory, vol. 41, no. 6, (2011), pp. 150-153. 
[5] W. Ke and B. Liu, "Efficient algorithm for constructing minimum size wireless sensor networks to fully cover critical square grids", IEEE Transactions on Wireless Communications, vol. 10, no. 4, (2011), pp. 1154-1164.

[6] S. Slijepcevic, M. Potkonjak, "Power efficient organization of wireless sensor networks", IEEE Conference on Communication, vol. 30, no. 2, (2013), pp. 472-476.

[7] B. Carbunar, A. Grama and J. Vitek, "Redundancy and Coverage Detection in Sensor Network", ACM Transactions on Sensor Network, vol. 30, no. 1, (2012), pp. 94-128.

[8] V. Mhatre and C. Rosenberg, "A Minimum cost heterogneous sensor network with a lifetime constraint”, IEEE Transaction on Mobile Computing, vol. 23, no. 3, (2012), pp. 27-31.

[9] N. Ruixin, K. Pramod and Q. Varshney, "Distributed Detection in a Large Wireless Sensor Network", Information Fusion, vol. 7, no. 4, (2006), pp. 380-384.

[10] Y. Jin and J. Jo, "An Energy Efficient Coverage and Connectivity Preserving Routing Algorithm under Border Effects in Wireless Senson Networks", Computer Communications, vol. 36, no. 10, (2012), pp. 2398-2407.

[11] Z. Jinhua, S. Zhongzhi and X A. Yong, "Fast Multi-Objective Genetic Algorithm Based on Clustering", Journal of Computer Research and Development, vol. 51, no. 7, (2014), pp. 10811087.

[12] Z. Sun and Y. Yu, "Coverage Control Algorithm Based on Ant Colony Algorithm in Wireless Sensor Networks", International Journal of Applied Mathematics and Statistics, vol. 50, no. 20, (2013), 1467-1475.

[13] H. Ammari and S. Das, "Centralized and Clustered K-coverage protocols for wireless sensor network", IEEE Transactions on Computers, vol. 61, no. 1, (2012), pp. 118-133.

[14] L. Liang and M. Huadong, "On Coverage of Wireless Sensor Networks for Rolling Terrains", IEEE Transactions on Parallel and Distributed Systems, vol. 8, no. 2, (2012), pp. 118-124.

[15] Y. Yonrim and K. Y. Hyuk, "An Efficient Genetic Algorithm for Maximum Coverage Deployment in Wireless Sensor Networks", IEEE Transactions on Cybernetics, vol. 10, no. 5, (2013), pp. 14731779.

[16] L. Liang, Z. Xi and M. Huang, "Percolation Theory-Based Exposure-Path Prevention for Wireless Sensor Networks Coverage in Internet of Things", IEEE Sensors Journal, vol. 12, no. 10,(2013),pp. 3625-3631.

[17] H. Chihfan and L. Ningyan, "Randomly Duty-cycled Wireless Sensor Networks: Dynamics of Coverage", IEEE Transactions on Wireless Communications, vol. 11, no. 5, (2006), pp. 3182-3188.

[18] B. Jacques, M. Abdallah and M. Ahmed, "Localization and Coverage for high density sensor networks", Computer and Communications, vol. 11, no. 5, (2012), pp. 770-776.

[19] M. Habib and K. Sajal, "Critical density for coverage and connectivity in Three-dimensional wireless sensor networks using continuum percolation", IEEE Transactions on Parallel and Distributed Systems, vol. 21, no. 6, (2009), pp. 872-881.

[20] D. Tian and N. Georganas, "A node scheduling scheme for energy conservation in large wireless sensor networks", Journal of Wireless Communications and Mobile Computing, vol. 12, no. 2, (2003), pp. 271-278.

[21] C. Hang, Y. Tseng and H. Wu, "Distributed protocols for ensuring both coverage and connectivity of a wireless sensor network", ACM Transactions on Sensor network, vol. 11, no. 1, (2007), pp. 137-145.

[22] S. Shakkottai, R. Srikant and N. Shroff, "Unreliable sensor grids: Coverage, Connectivity and Diameter", Ad Hoc Network, vol. 7, no. 4, (2005), pp. 702-716.

[23] P. Zhou, X. Cui and S. Wang, "Virtual force based wireless sensor network coverage enhancing algorithm", Journal of system simulation, vol. 10, no. 9, (2009), pp. 1416-1422. 
International Journal of Future Generation Communication and Networking Vol. 9, No. 5 (2016) 